\title{
Predictive relevance of lymphovascular invasion in T1 colorectal cancer before endoscopic treatment
}

\section{(ㄷ)(1) $\Theta$}

Authors

Kazuya Inoki ${ }^{1,4}$, Taku Sakamoto ${ }^{1}$, Hiroyuki Takamaru ${ }^{1}$, Masau Sekiguchi ${ }^{1}$, Masayoshi Yamada $^{1}$, Takeshi Nakajima $^{1}$, Takahisa Matsuda ${ }^{1}$, Hirokazu Taniguchi ${ }^{2}$, Shigeki Sekine ${ }^{2}$, Yukihide Kanemitsu ${ }^{3}$, Yuichiro Ohe ${ }^{4}$, Yutaka Saito ${ }^{1}$

Institutions

1 Endoscopy Division, National Cancer Center Hospital, Tokyo, Japan

2 Pathology and Clinical Laboratory Division, National Cancer Center Hospital, Tokyo, Japan

3 Colorectal Surgery Division, National Cancer Center Hospital, Tokyo, Japan

4 Course of Advanced Clinical Research of Cancer, Juntendo University Graduate School of Medicine, National Cancer Center Hospital, Tokyo, Japan

submitted 3.1.2017

accepted after revision 26.6.2017

\section{Bibliography}

DOI https://doi.org/10.1055/s-0043-117952 |

Endoscopy International Open 2017; 05: E1278-E1283

(c) Georg Thieme Verlag KG Stuttgart · New York

ISSN 2364-3722

Corresponding author

Taku Sakamoto, MD, National Cancer Center Hospital, 5-1-1 Tsukiji, Chuo-ku, Tokyo, 104-0045, Japan

Fax: $+81-3-35423815$

tasakamo@ncc.go.jp

\section{ABSTRACT}

Background and aim The depth of tumor invasion is currently the only reliable predictive risk factor for lymph node metastasis before endoscopic treatment for colorectal can- cer. However, the most important factor to predict lymph node metastasis has been suggested to be lymphovascular invasion rather than the depth of invasion. Thus, the aim of this study was to investigate the predictive relevance of lymphovascular invasion before endoscopic treatment.

Methods The data on PT1 colorectal cancers that were resected endoscopically or surgically from 2007 to 2015 were retrospectively reviewed. The cases were categorized into two groups: positive or negative for lymphovascular invasion. The following factors were evaluated by univariate and multivariate analyses: age and sex of the patients; location, size, and morphology of the lesion; and depth of invasion.

Results The positive and negative groups included 229 and 457 cases, respectively. Younger age $(P<0.01)$, smaller lesion size $(P=0.01)$, non-LST (LST: laterally spreading tumor) $(P<0.01)$, presence of depression $(P<0.01)$, and pT1b $(P<$ $0.01)$ were associated with lymphovascular invasion. In multivariate analysis, younger age (comparing patients aged $\leq 64$ years with those aged $>65$ years, OR, 1.81; $95 \%$ $\mathrm{Cl}, 1.29-2.53)$, presence of depression (OR, 1.97; Cl, $1.40-2.77$ ), non-LST features (OR, 1.50; Cl, 1.04-2.15), and pT1b (OR, 3.08; $\mathrm{Cl}, 1.91-4.97)$ were associated with lymphovascular invasion.

Conclusion Younger age, presence of depression, T1b, and non-LST are associated with lymphovascular invasion. Therefore, careful pathological diagnosis and surveillance are necessary for lesions demonstrating any of these four factors.

\section{Introduction}

Endoscopic resection (ER) for early stage colorectal tumors is now a widely accepted type of treatment. The recent development of endoscopic submucosal dissection (ESD) has enabled us to perform en bloc resection of large lesions that were previously resected in piecemeal fashion, thereby decreasing the local recurrence rate [1-4]. However, the application of ER is mostly limited to lesions with a negligible risk of lymph node metastasis (LNM) $[5,6]$, since lymph node dissection is not per- formed using this method. Therefore, in addition to an appropriate preoperative diagnosis using high definition endoscopy and chromoendoscopy, a precise evaluation of post-ER pathological findings and risk stratification is necessary to assess the risk of LNM and evaluate patients for appropriate additional treatment and surveillance programs [5].

According to the Japanese Society for Cancer of the Colon and Rectum (JSCCR) Guidelines, the curability of the tumor can be estimated by pathological factors. If the lesion is invasive, negative vertical and horizontal margins, as well as the fol- 
lowing four factors, are required for a resection to be considered curative: (1) depth of submucosal invasion less than 1000 $\mu \mathrm{m},(2)$ well and/or moderately differentiated adenocarcinoma, (3) negative lymphovascular invasion (LVI), (4) budding grade 1. If any of these factors are not fulfilled, additional surgery should be considered to reduce the risk of recurrent LNM and mortality [5]. The European Society of Gastrointestinal Endoscopy (ESGE) Guidelines [7] use similar criteria to evaluate the curability of endoscopically resected specimens. In addition to those factors, the ESGE Guidelines mention the importance of evaluating budding, and recommend at least a $1 \mathrm{~mm}$ tumorfree margin to obtain curative resection. Although the American Society for Gastrointestinal Endoscopy (ASGE) Guidelines [8] do not mention any pathological criteria after ER, the National Comprehensive Cancer Network (NCCN) Guidelines have pathological criteria that are similar to those of JSCCR and ESGE.

Both Japanese and Western guidelines for ER of colorectal tumors have similar criteria to evaluate the curability of resected specimen as well. Of several factors influencing the curability, the depth of tumor invasion, which can be measured with high accuracy by magnifying endoscopy, is currently the only reliable predictive risk factor before endoscopic treatment of colorectal cancer (CRC). However, LVI or histological type has been suggested as an even more important predictor of lymph node metastasis and poor prognosis [9-15]. So far, the endoscopic findings that correlate with LVI have not been elucidated.

The aim of this study is to investigate the predictive relevance of LVI before endoscopic treatment.

\section{Patients/Materials and methods}

Electronic medical records and pathological reports of patients who underwent ER or surgical resection (SR) at the National Cancer Center, Tokyo, Japan, between April 2007 and April 2015 were retrospectively reviewed. The clinical and pathological outcomes of 679 consecutive patients with 703 pT1 colorectal neoplasms were gathered. We investigated relevant factors that could be obtained or predicted with high accuracy before endoscopic treatment, including age, sex, tumor location (lower rectum, upper rectum, or colon), size and morphology of the lesion by review of endoscopic reports. On the other hand, the data on size and depth of invasion were obtained by review of pathological reports. Thirteen patients with 13 lesions were excluded due to difficulty determining whether the tumors were pathologically T1a or T1b. In addition, one patient with four lesions was excluded since it was difficult to evaluate tumor morphology due to the collapse of the lesions. Finally, 665 patients with a total of 686 pT1 colorectal neoplasms were analyzed.

The patients/lesions were divided into two groups, a LVI positive group and a LVI negative group ( $\triangleright$ Fig. 1 ). The morphology of the lesion includes determining the presence of depressions and laterally spreading tumors (LST). The depth of invasion was divided into two groups, T1a (depth of invasion $\leq 1000 \mu \mathrm{m})$ and T1b (depth of invasion $>1000 \mu \mathrm{m}$ ), in accord-
679 patients with 703 pT1 colorectal cancers that were resected endoscopically or surgically from April 2007 to April 2015

Exclusion:

- 13 patients with 13 lesions that were difficult to diagnose as PT1a or PT1b

- 1 patient with 4 lesions where it was difficult to determine the morphology due to collapse of the lesion

\begin{tabular}{|c|c|c|c|}
\hline \multicolumn{2}{|c|}{$\sqrt{x}$} & \multicolumn{2}{|c|}{$\downarrow$} \\
\hline \multicolumn{2}{|c|}{$\begin{array}{l}\text { ER } \\
314 \text { patients } 322 \text { lesions }\end{array}$} & \multicolumn{2}{|c|}{$\begin{array}{l}\text { SR } \\
351 \text { patients } 364 \text { lesions }\end{array}$} \\
\hline$\sqrt{ }$ & $\downarrow$ & $\sqrt{ }$ & $\downarrow$ \\
\hline $\begin{array}{l}\mathrm{LVI}+ \\
79 \text { lesions }\end{array}$ & $\begin{array}{l}\text { LVI - } \\
243 \\
\text { lesions }\end{array}$ & $\begin{array}{l}\text { LVI + } \\
150 \\
\text { lesions }\end{array}$ & $\begin{array}{l}\text { LVI - } \\
214 \\
\text { lesions }\end{array}$ \\
\hline$\downarrow$ & & & $\downarrow$ \\
\hline $\begin{array}{l}\mathrm{LVI}+ \\
229 \text { lesions }\end{array}$ & & $\begin{array}{l}\text { LVI - } \\
457 \text { lesi }\end{array}$ & \\
\hline
\end{tabular}

$E R$, endoscopic resection; SR, surgical resection; LVI, lymphovascular invasion

- Fig. 1 Inclusion and exclusion criteria of patients. Lesions are divided into two groups according to the presence (LVI+) or absence (LVI-) of lymphovascular invasion.

ance with the Japanese classification criteria for cancer of the colon and rectum. Pathological depth (pT1a, pT1b) was used instead of clinical depth (cT1a, cT1b), because the accuracy of the depth diagnosis of colorectal lesions using magnifying chromoendoscopy is reported to be $>90 \%$ [16]. We conducted this study in accordance with the guidelines of our Institutional Review Board, which approved this retrospective study without the need for informed consent. All of the patients provided written informed consent for the ER or SR.

\section{Histopathological assessment}

Endoscopically resected specimens were fixed in $10 \%$ buffered formalin and were cut into 2 -mm-thick slices. In contrast, surgical specimens were cut into 4- to 5-mm-thick slices. These were embedded in paraffin, cut into $3-\mu \mathrm{m}$ sections, stained with hematoxylin and eosin ( $\mathrm{HE}$ ), and microscopically examined for histopathologic type by pathologists specialized in gastrointestinal pathology. The histopathological diagnoses were based on the Japanese classification criteria for cancer of the colon and rectum. In addition to HE staining, podoplanin (D2 -40) and Elastica van Gieson (EVG) staining were performed for the evaluation of lymphatic invasion and venous invasion, respectively, with endoscopically resected specimens. In contrast, for surgical specimens, Victoria Blue hematoxylin and eosin (VB-HE) staining was performed to assess venous invasion, however, po- 
doplanin staining was not performed. Lymphovascular invasion was determined to be positive when tumor cells were observed in lymphatic or vascular spaces.

\section{Statistical analysis}

To examine the relevance of LVI as a predictive marker of curability, age, sex, tumor location (rectum, colon), size and morphology of the lesion (presence of depression, LST), and depth of invasion (pT1a, pT1b) were evaluated. For comparisons between the LVI positive and negative groups, data were analyzed using the Mann-Whitney $U$ test for data showing continuous variables and Fisher's exact test for categorical variables. Conditional multivariate logistic regression analysis using all variables was constructed to calculate odds ratios (ORs) and $95 \%$ confidence intervals $(\mathrm{Cl})$. All tests were two-sided, and $P<0.05$ was considered to be statistically significant. All statistical analyses were performed with EZR (Saitama Medical Center, Jichi Medical University, Saitama, Japan), which is a graphical user interface for R (The R Foundation for Statistical Computing, Vienna, Austria). More precisely, it is a modified version of $R$ Commander designed to add statistical functions frequently used in biostatistics [17].

\section{Results}

Our study included samples from 314 patients with 322 lesions who had ER and samples from 351 patients with 364 lesions who had SR ( Fig. 1; Table 1). Of 322 ER lesions, 302 cases were resected en bloc and 20 lesions were resected in piecemeal fashion. The proportion of female subjects was lower in ER than SR (35.4\% vs $44.0 \%$, respectively; $P=0.02)$. A significantly lower proportion of tumor samples from the ER group versus the SR group had depressed components $(30.7 \%$ vs $65.7 \%$, respectively; $P<0.01)$, non-LST features $(46.6 \%$ vs $71.3 \%$, respectively; $P<0.01$ ), and LVI ( $32.5 \%$ vs $41.2 \%$, respectively; $P<0.01)$. In addition, a significantly lower proportion of samples from the ER group than the SR group was diagnosed as T1b (52.8\% vs $94.0 \%$, respectively; $P<0.01)$. No significant differences were found between ER and SR groups with regard to age, tumor location, or tumor size ( $\vee$ Table 1 ).

LVI positive and negative groups included 229 and 457 cases, respectively ( $\triangleright$ Fig. 1 ; $>$ Table 2 ). Age was significantly lower in patients in the LVI positive group than the LVI negative group (65 years vs 67 years, respectively; $P<0.01$ ), and median tumor size was significantly smaller in the LVI positive group than the LVI negative group ( $17 \mathrm{~mm}$ vs $20 \mathrm{~mm}$, respectively; $P$ $=0.01$ ). A significantly higher proportion of samples from the LVI positive group than the LVI negative group had depressed components ( $62.9 \%$ vs $42.5 \%$, respectively; $P<0.01$ ), non-LST features $(71.2 \%$ vs $55.8 \%$, respectively; $P<0.01)$, and $\mathrm{pT} 1 \mathrm{~b}$ $(89.0 \%$ vs $67.4 \%$, respectively; $P<0.01)$ ( $>$ Table 2$)$. In a multivariate logistic regression analysis, younger age (comparing patients aged $\leq 64$ years with those aged $>65$ years; OR: $1.81 ; 95 \%$ $\mathrm{Cl}, 1.29-2.53$ ), depressed component (OR: 1.97; Cl, $1.40-$ 2.77), non-LST feature (OR: $1.50 ; \mathrm{Cl}, 1.04-2.15$ ), and pT1b (OR: $3.08 ; \mathrm{Cl}, 1.91-4.97$ ) were shown to be associated with LVI ( $\triangleright$ Table 3). Approximately half of the lesions (47.5\%) in
- Table 1 Clinical characteristics of T1 colorectal cancer cases treated with ER or SR.

\begin{tabular}{|c|c|c|c|}
\hline Characteristics & $E R(n=322)$ & $S R(n=364)$ & $P$ value \\
\hline Age, median (IQR) & $68(60-74)$ & $67(59-73)$ & 0.57 \\
\hline \multicolumn{4}{|l|}{ Sex } \\
\hline - Male & $208(64.6 \%)$ & $204(56.0 \%)$ & 0.02 \\
\hline - Female & $114(35.4 \%)$ & $160(44.0 \%)$ & \\
\hline \multicolumn{4}{|l|}{ Location } \\
\hline - Rectum & $67(20.8 \%)$ & $59(16.2 \%)$ & 0.14 \\
\hline - Other locations & $255(79.2 \%)$ & $305(83.8 \%)$ & \\
\hline Size, median (IQR) & $20(12-29)$ & $18(12-25)$ & 0.28 \\
\hline \multicolumn{4}{|l|}{ Morphology } \\
\hline - Depression (+) & $99(30.7 \%)$ & $239(65.7 \%)$ & $<0.01$ \\
\hline - Depression (-) & $223(69.3 \%)$ & $125(34.3 \%)$ & \\
\hline . LST & $172(53.4 \%)$ & $96(28.7 \%)$ & $<0.01$ \\
\hline - Non-LST & $150(46.6 \%)$ & $238(71.3 \%)$ & \\
\hline \multicolumn{4}{|l|}{ Depth of invasion } \\
\hline - T1a & $152(47.2 \%)$ & $22(6.0 \%)$ & $<0.01$ \\
\hline - $\mathrm{T} 1 \mathrm{~b}$ & $170(52.8 \%)$ & $342(94.0 \%)$ & \\
\hline \multicolumn{4}{|l|}{ LVI } \\
\hline - Positive & $79(32.5 \%)$ & $150(41.2 \%)$ & $<0.01$ \\
\hline - Negative & $243(67.5 \%)$ & $214(58.8 \%)$ & \\
\hline \multicolumn{4}{|l|}{ Status of ER } \\
\hline - En bloc & $302(93.8 \%)$ & & \\
\hline - Piecemeal fashion & $20(6.2 \%)$ & & \\
\hline
\end{tabular}

samples with a depressed component and suspected $\mathrm{T} 1 \mathrm{~b}$, were LVI positive ( $\triangleright$ Table 4$)$.

\section{Discussion}

The histological identification of LVI after colorectal cancer (CRC) removal has been recognized as a reliable predictor of LNM and patient prognosis. This study investigated the predictive relevance of LVI before endoscopic treatment and showed two clinically important issues. First, younger age, depression, T1b, and non-LST are associated with LVI. Second, careful pathological diagnosis and surveillance are necessary for lesions demonstrating any of these four factors. In addition to depth diagnosis, recognition of depressed components and non-LST status by endoscopic diagnosis is important to predict LVI.

Our study suggests that T1 CRCs in younger patients may have more malignant potential than those in older individuals. There is growing evidence that the incidence of CRC in the 
Table 2 Univariate analysis to predict LVI status using factors that can be acquired during endoscopic diagnosis.

\begin{tabular}{|c|c|c|c|}
\hline Characteristics & LVI positive $(n=229)$ & LVI negative $(n=457)$ & $P$ value \\
\hline Age, median (IQR) & $65(57-72)$ & $67(61-74)$ & $<0.01$ \\
\hline \multicolumn{4}{|l|}{ Sex } \\
\hline - Male & $144(62.9 \%)$ & $268(58.6 \%)$ & 0.32 \\
\hline - Female & $85(37.1 \%)$ & $189(41.4 \%)$ & \\
\hline \multicolumn{4}{|l|}{ Location } \\
\hline - $\mathrm{Rb}$ & $48(21.0 \%)$ & $78(17.1 \%)$ & 0.25 \\
\hline - Other locations & $181(79.0 \%)$ & $379(82.9 \%)$ & \\
\hline Size, median (IQR) & $17(12-24)$ & $20(12-28)$ & 0.01 \\
\hline \multicolumn{4}{|l|}{ Morphology } \\
\hline - Depression (+) & $144(62.9 \%)$ & $194(42.5 \%)$ & $<0.01$ \\
\hline - Depression (-) & $85(37.1 \%)$ & $263(57.5 \%)$ & \\
\hline " LST & $66(28.8 \%)$ & $202(44.2 \%)$ & $<0.01$ \\
\hline - Non-LST & $163(71.2 \%)$ & $255(55.8 \%)$ & \\
\hline \multicolumn{4}{|l|}{ Depth of invasion } \\
\hline - T1a & $25(11.0 \%)$ & $149(32.6 \%)$ & $<0.01$ \\
\hline " T1b & 204 (89.0\%) & 308 (67.4\%) & \\
\hline
\end{tabular}

- Table 3 Multivariate analysis to predict LVI status using factors that can be acquired during endoscopic diagnosis.

\begin{tabular}{|c|c|c|}
\hline Clinical factors & OR $(95 \% \mathrm{Cl})$ & $P$ value \\
\hline \multicolumn{3}{|l|}{ Age } \\
\hline . $>65$ & 1.00 (ref) & $<0.01$ \\
\hline - $\leq 65$ & $1.81(1.29-2.53)$ & \\
\hline \multicolumn{3}{|l|}{ Morphology } \\
\hline - Depression (-) & 1.00 (ref) & $<0.01$ \\
\hline - Depression (+) & $1.97(1.40-2.77)$ & \\
\hline - LST & 1.00 (ref) & 0.03 \\
\hline - Non-LST & $1.50(1.04-2.15)$ & \\
\hline \multicolumn{3}{|l|}{ Depth of invasion } \\
\hline - T1a & 1.00 (ref) & $<0.01$ \\
\hline - $\mathrm{T} 1 \mathrm{~b}$ & $3.08(1.91-4.97)$ & \\
\hline
\end{tabular}

younger generation is increasing $[18,19]$, and it has been suggested that CRC in younger patients exhibits a different biological behavior than in older patients [20-22]. Although it still remains controversial, some studies have shown that younger patients with CRC have a worse prognosis [23,24]. Our study supports these results; however, further examination is necessary to confirm our conclusion. Furthermore, our study showed
- Table 4 Rate of lymphovascular invasion by depth of invasion or depression.

\begin{tabular}{|l|l|r|}
\hline & \multicolumn{2}{|l|}{ Depth of invasion } \\
\hline & T1a & \multicolumn{1}{|c|}{ T1b } \\
\hline Morphology & & \\
\hline - Depression (-) & $15 / 118(12.7 \%)$ & $70 / 230(30.4 \%)$ \\
\hline - Depression (+) & $10 / 56(17.9 \%)$ & $134 / 282(47.5 \%)$ \\
\hline
\end{tabular}

that T1b colorectal cancers tend to have a higher rate of LVI than T1a colorectal cancers. This is probably because T1b cancers have more malignant potential than T1a cancer and a larger invasive area raises the possibility of encountering lymphatic ducts or small vessels.

Our study also suggests that depression and non-LST lesions are associated with LVI. These results may reflect the typical characteristics of de novo cancer such as depressed type lesions or non-polypoid growth $[25,26]$. Cancers derived from the de novo pathway are considered more aggressive than those derived from an adenoma carcinoma sequence [27]. For well-trained endoscopists, determining the presence of depressions, the depth of the tumor, and LST with high confidence is possible endoscopically [16]. Endoscopists should carefully examine these findings and make a precise diagnosis during pretreatment endoscopy. These three factors are also present in typical submucosal invasive cancer that has a definite depres- 
sed area with distinct margins: 0 -IIc or 0 -IIa + IIc or 0 -Is + IIc lesion.

Recently, expanding the criteria for endoscopic resection for T1b colorectal cancers has been discussed, and there is a possibility that ER for T1b colorectal cancers could become standard treatment if other factors are fulfilled $[28,29]$. In a retrospective study, Yoshii et al. demonstrated that patients who underwent ER for deep submucosal invasive cancer are at low risk for colon cancer recurrence if the there are no lymphovascular invasion, poor differentiation, and high grade budding [29]. They estimated the cumulative risk of recurrence to be $2.3 \%$ in an ER plus additional surgery group and $3.4 \%$ in an ER only group $(P=$ 0.867). However, they experienced one death after ER due to recurrence in a patient who was diagnosed as pT1b $(2300 \mu \mathrm{m})$ with no other non-curative factors after ER.

Considering the fact that even though a lesion is cut into 2 $\mathrm{mm}$ slices when performing the pathological diagnosis for ER specimens in Japan, not all areas of a lesion are observed pathologically, and it is impossible to observe the entire lesion continuously. A substantial risk may exist that LVI positivity is not diagnosed pathologically. It was reported that interobserver agreement for LVI among pathologists is insufficient for a credible diagnosis, even if immunohistochemical staining was used [30]. That is the main limitation of the current pathological diagnosis. In fact, recurrent cases of colon cancer are occasionally experienced in cases where the lesion was diagnosed as LVI negative during initial diagnosis, but was shown to be positive after reevaluation with additional more deeply cut specimens that were performed when the recurrence was recognized. We must recognize from this study that $\mathrm{T} 1 \mathrm{~b}$ itself is a risk factor for LVI positivity, and pathological evaluation should be performed in a standardized sophisticated manner by experienced gastrointestinal pathologists. Additionally, prudent discussion is necessary when considering expanding the criteria for ER of T1b colorectal cancers. When ER is performed for a lesion that shows a depression, PT1b, and non-LST, there is a possibility that LVI was missed, even if the lesion was diagnosed initially as LVI negative.

The accuracy of the diagnosis of LVI in our study was not sufficiently high to change the endoscopic treatment strategy for early colorectal cancers. On the contrary, the results from our study suggest that if the lesion demonstrates younger age, a depressed component, T1b, and non-LST, careful pathological diagnosis is necessary to predict treatment outcomes. In addition, an endoscopist should carefully diagnose the morphology, depression, and estimated depth using high definition endoscopy and chromoendoscopy.

This study has several limitations. In our study, we investigated the predictive factors from endoscopic diagnosis; however, we used the pathological depth data for our investigation. Since the accuracy of the endoscopic diagnosis is satisfactory, we reasoned that substituting the endoscopically estimated depth measurement with the pathological depth measurement is acceptable. Furthermore, there are several differences about pathological diagnosis between endoscopic and surgical specimens. First, endoscopically resected specimens were cut into 2mm-thick slices. In contrast, surgical specimens were cut into
4- to 5-mm-thick slices. This may influence the positive ratio of LVI. Second, EVG staining was used for endoscopically resected specimens, whereas VB-HE staining was used for surgical specimens to evaluate vascular invasion. Although there is a difference concerning staining of vessels, and both stains are considered to be useful [31-33], it is unlikely that this might influence the positive ratio of venous invasion. Third, D2-40 staining was not performed for SR, because lymph node dissection had already been performed. This might underestimate the presence of lymphatic invasion when we evaluated the LVI status in surgical specimens.

In conclusion, younger age, depressed component, T1b, and non-LST are associated with LVI. Careful pathological diagnosis is necessary to predict treatment outcomes if any of these four factors are present. Further multicenter or prospective studies are necessary to determine the endoscopic features from which we can predict LVI.

\section{Acknowledgments}

The authors thank Dr. Saowanee Ngamruengphong (Division of Gastroenterology \& Hepatology, Johns Hopkins Medicine, Baltimore, MD, United States) for constructive comments and editing of the manuscript.

This study was conducted with a Grant-in-Aid from the Japan Agency for Medical Research and Development, AMED (16ck0106028h0003).

\section{Competing interests}

None

References

[1] Kobayashi N, Yoshitake N, Hirahara Y et al. Matched case-control study comparing endoscopic submucosal dissection and endoscopic mucosal resection for colorectal tumors. J Gastroenterol Hepatol 2012; 27: $728-733$

[2] Nakajima T, Saito Y, Tanaka S et al. Current status of endoscopic resection strategy for large, early colorectal neoplasia in Japan. Surg Endosc 2013; 27: $3262-3270$

[3] Oka S, Tanaka S, Saito Y et al. Local recurrence after endoscopic resection for large colorectal neoplasia: a multicenter prospective study in Japan. Am J Gastroenterol 2015; 110: 697-707

[4] Sakamoto T, Mori G, Yamada M et al. Endoscopic submucosal dissection for colorectal neoplasms: a review. World J Gastroenterol 2014; 20: $16153-16158$

[5] Watanabe T, Itabashi M, Shimada Y et al. Japanese Society for Cancer of the Colon and Rectum (JSCCR) Guidelines 2014 for treatment of colorectal cancer. Int J Clin Oncol 2015; 20: 207-239

[6] Saito Y, Sakamoto T, Nakajima T et al. Colorectal ESD: current indications and latest technical advances. Gastrointest Endosc Clin North Am 2014; 24: $245-255$

[7] Pimentel-Nunes P, Dinis-Ribeiro M, Ponchon T et al. Endoscopic submucosal dissection: European Society of Gastrointestinal Endoscopy (ESGE) Guideline. Endoscopy 2015; 47: 829-854 
[8] Fisher DA, Shergill AK, Early DS et al. Role of endoscopy in the staging and management of colorectal cancer. Gastrointest Endosc 2013; 78: $8-12$

[9] Lim SB, Yu CS, Jang S] et al. Prognostic significance of lymphovascular invasion in sporadic colorectal cancer. Dis Colon Rectum 2010; 53 : $377-384$

[10] Fang WL, Chang SC, Lin JK et al. Metastatic potential in T1 and T2 colorectal cancer. Hepatogastroenterology 2005; 52: 1688 - 1691

[11] Akagi Y, Adachi Y, Ohchi T et al. Prognostic impact of lymphatic invasion of colorectal cancer: a single-center analysis of 1,616 patients over 24 years. Anticancer Res 2013; 33: 2965 - 2970

[12] Meining A, von Delius S, Eames TM et al. Risk factors for unfavorable outcomes after endoscopic removal of submucosal invasive colorectal tumors. Clin Gastroenterol Hepatol 2011; 9: 590 - 594

[13] Glasgow SC, Bleier JI, Burgart L] et al. Meta-analysis of histopathological features of primary colorectal cancers that predict lymph node metastases. J Gastrointest Surg 2012; 16: 1019-1028

[14] Beaton C, Twine CP, Williams GL et al. Systematic review and meta-analysis of histopathological factors influencing the risk of lymph node metastasis in early colorectal cancer. Colorectal Dis 2013; 15: 788 797

[15] Kim B, Kim EH, Park S] et al. The risk of lymph node metastasis makes it unsafe to expand the conventional indications for endoscopic treatment of T1 colorectal cancer: A retrospective study of 428 patients. Medicine 2016; 95: e4373

[16] Matsuda T, Fujii T, Saito Y et al. Efficacy of the invasive/non-invasive pattern by magnifying chromoendoscopy to estimate the depth of invasion of early colorectal neoplasms. Am J Gastroenterol 2008; 103: $2700-2706$

[17] Kanda Y. Investigation of the freely available easy-to-use software 'EZR' for medical statistics. Bone Marrow Transplant 2013; 48: 452 458

[18] Austin H, Henley SJ, King J et al. Changes in colorectal cancer incidence rates in young and older adults in the United States: what does it tell us about screening. Cancer Causes Control 2014; 25: 191-201

[19] Young JP, Win AK, Rosty C et al. Rising incidence of early-onset colorectal cancer in Australia over two decades: report and review. J Gastroenterol Hepatol 2015; 30: 6-13

[20] Liang JT, Huang KC, Cheng AL et al. Clinicopathological and molecular biological features of colorectal cancer in patients less than 40 years of age. Br J Surg 2003; 90: 205-214
[21] Tezcan G, Tunca B, Ak S et al. Molecular approach to genetic and epigenetic pathogenesis of early-onset colorectal cancer. World J Gastrointest Oncol 2016; 8: 83-98

[22] Silla IO, Rueda D, Rodriguez Y et al. Early-onset colorectal cancer: a separate subset of colorectal cancer. World J Gastroenterol 2014; 20: $17288-17296$

[23] Zbuk K, Sidebotham EL, Bleyer A et al. Colorectal cancer in young adults. Semin Oncol 2009; 36: 439-450

[24] Chan KK, Dassanayake B, Deen R et al. Young patients with colorectal cancer have poor survival in the first twenty months after operation and predictable survival in the medium and long-term: analysis of survival and prognostic markers. World J Surg Oncol 2010; 8: 82

[25] lishi H, Tatsuta M, Tsutsui S et al. Early depressed adenocarcinomas of the large intestine. Cancer 1992; 69: 2406-2410

[26] Kudo S, Kashida H, Tamura T. Early colorectal cancer: flat or depressed type. J Gastroenterol Hepatol 2000; 15 Suppl: D66 - 70

[27] Kudo S, Tamure S, Nakajima T et al. Depressed type of colorectal cancer. Endoscopy 1995; 27: 54 - 57; discussion 61

[28] Nakadoi K, Tanaka S, Kanao H et al. Management of T1 colorecta carcinoma with special reference to criteria for curative endoscopic resection. J Gastroenterol Hepatol 2012; 27: 1057 -1062

[29] Yoshii S, Nojima M, Nosho K et al. Factors associated with risk for colorectal cancer recurrence after endoscopic resection of T1 tumors. Clin Gastroenterol Hepatol 2014; 12: 292 -302.e2

[30] Harris El, Lewin DN, Wang HL et al. Lymphovascular invasion in colorectal cancer: an interobserver variability study. Am J Surg Pathol 2008; 32: $1816-1821$

[31] Tsutsumi Y, Onoda N, Osamura RY. Victoria blue-hematoxylin and eosin staining: a useful routine stain for demonstration of venous invasion by cancer cells. J Histotechnol 1990; 13: 271 - 274

[32] Inoue T, Mori M, Shimono R et al. Vascular invasion of colorectal carcinoma readily visible with certain stains. Dis Colon Rectum 1992; 35: $34-39$

[33] Suzuki A, Togashi K, Nokubi M et al. Evaluation of venous invasion by Elastica van Gieson stain and tumor budding predicts local and distant metastases in patients with T1 stage colorectal cancer. Am J Surg Pathol 2009; 33: 1601 - 1607 